\title{
Tracking the progress of HIV: the impact of point-of-care tests on antiretroviral therapy
}

This article was published in the following Dove Press journal:

Clinical Epidemiology

30 September 2013

Number of times this article has been viewed

\section{Steven D Reid \\ Sarah J Fidler \\ Graham S Cooke}

Department of Infectious Diseases, St Mary's Hospital, Imperial College London, London, UK
Correspondence: Steven D Reid CD4 Initiative, Imperial College London, The Bays, South Wharf Road, St Mary's Hospital, London, W2 INY, UK

Tel +442033122563

Email s.d.reid@imperial.ac.uk
Abstract: It is now around 30 years since the discovery of HIV, the virus that causes AIDS. More than 70 million people have been infected in that time and around 35 million have died. The majority of those currently living with HIV/AIDS are in low- and middle-income countries, with sub-Saharan Africa bearing a disproportionate burden of the global disease. In high-income countries, the introduction of antiretroviral therapy (ART) has drastically reduced the morbidity and mortality associated with HIV. Patients on ART are now predicted to have near-normal life expectancy and the role of treatment is increasingly recognized in preventing new infections. In low- and middle-income countries, treatment is now more widely available and around half of those who need ART are currently receiving it. Early diagnosis of HIV is essential if ART is to be optimally implemented. Lab-based diagnostics for screening, diagnosis, treatment initiation, and the monitoring of treatment efficacy are critical in managing the disease and reducing the number of new infections each year. The introduction of point-of-care HIV rapid tests has transformed the epidemic, particularly in low- and middle-income countries. For the first time, these point-of-care tests allow for the rapid identification of infected individuals outside the laboratory who can undergo counseling and treatment and, in the case of pregnant women, allow the timely initiation of ART to reduce the risk of vertical transmission. Although survival is markedly improved with ART even in the absence of laboratory monitoring, long-term management of people living with HIV on ART, and their partners, is essential to ensure successful viral suppression. The burden of disease in many resource-poor settings with high HIV prevalence has challenged the ability of local laboratories to effectively monitor those on ART. Diagnostics used to initiate and monitor treatment are now moving out of the laboratory and into the field. These new point-of-care tests for viral load and CD4 are poised to further transform the disease and shift the treatment paradigm in low- and middle-income countries.

Keywords: point-of-care, diagnostics, HIV, CD4, viral load, resource-poor countries

\section{Introduction}

Much progress has been made in stemming the HIV/AIDS pandemic; however, there are currently around 34 million people living with HIV/AIDS globally. ${ }^{1}$ The majority of those living with the disease are in sub-Saharan Africa, where an estimated 23 million people are infected. Recent global epidemiological data indicate that there were fewer new infections in 2011 (2.5 million) than 10 years ago (3.1 million). The introduction of antiretroviral therapy (ART) has saved millions of lives, and the number of deaths each year due to HIV is decreasing, from a high in 2006 at 2.3 million to the current 1.7 million (Table 1). These data have led to growing hope that the pandemic has peaked. In high-income countries, ART and the accompanying diagnostics used 
Table I Epidemiological data for HIV infections in 200 I and 20I I

\begin{tabular}{lll}
\hline & 200I & 20I I \\
\hline Total living with HIVIAIDS & 29.4 million & 34 million \\
New infections & 1.9 million & 1.7 million \\
Deaths & 3.2 million & 2.5 million \\
\hline
\end{tabular}

Notes: Reproduced with permission from World Health Organization. HIV/ AIDS: data and statistics. Available from: http://www.who.int/hiv/data/en/index.html. Accessed April 26, 2013.'

in HIV care are readily available. In contrast, in low- and middle-income countries (LMICs), there is often limited access to these essential drugs and diagnostic tests.

In resource-constrained settings with large rural populations, health care is accessed primarily at local health centers. These centers often have only rudimentary services - many operate without running water or constant power and so only relatively simple procedures and diagnostic tests are available. There are no or few trained laboratory technicians and the clinics are most often nurse-led. Due to the size of the HIV epidemic and the lack of infrastructure in LMICs, these health centers are vital in managing those with HIV/AIDS. While there has been much success in the implementation of ART in these settings, the World Health Organization (WHO) estimates that just over half of the 11 million people requiring treatment in sub-Saharan Africa are actually receiving it. ${ }^{1}$

\section{Diagnostics and HIV: HIV testing and the shift to point-of-care (POC)}

After the discovery and characterization of HIV in the early 1980 s, there was an urgent need to develop diagnostic tests. ${ }^{2,3}$ Early cases of HIV were almost always fatal: patients succumbed to opportunistic infections like Pneumocystis pneumonia or Kaposi's sarcoma because HIV primarily infects CD4-bearing T lymphocytes, which are essential for a functioning immune system. The first assays looked for the presence of antibodies to HIV in the patient's blood and not the pathogen itself., 4 These enzyme-linked immunosorbent assays (ELISA) for HIV were the first to be developed commercially for screening and diagnosis. Due to the nature of the infection and the sensitivity of these early assays, infection could not be confirmed for up to 3 months after exposure. Despite this lag time, these assays were essential for diagnosis and for the screening of donor blood samples. ${ }^{6}$ Confirmatory assays were added, such as western blot, to improve the assay specificity, particularly for the earlier iterations of the ELISA. These assays have undergone extensive revision with markedly improved sensitivity and specificity. However, both techniques are relatively labor-intensive, and the high-throughput ELISA required significant instrument costs, even if the "per-test" cost per sample was relatively low. In LMICs, these tests were vital in understanding the emerging epidemic, but they required sample transport to centralized laboratory services and a relatively sophisticated referral system. As the world slowly became aware of the epidemic of HIV in Africa, it was clear that implementing testing processes found in high-income countries was not possible. $^{7}$

POC, or rapid tests, were developed in the 1990s and were aimed at testing markets without access to laboratory services. The most successful of these was the lateral flow assay and the impact of this POC test on the HIV pandemic cannot be underestimated. For the first time, health care centers were able to offer rapid HIV testing (under 1 hour), albeit with a combination of rapid tests and an algorithm to ensure high sensitivity and specificity. ${ }^{8}$ These patients could then be triaged to treatment more effectively. This was particularly important in diagnosing pregnant women when even a one-off dose of ART at the time of delivery could dramatically reduce the chance of HIV transmission to the infant. ${ }^{9}$ Importantly, these rapid POC tests were able to prevent much of the loss to follow-up seen in LMICs, as patients did not have to wait 1-2 weeks for the results of the assay. In 2004, the WHO issued evaluations of POC HIV tests commercially available for screening and diagnosis. ${ }^{10}$

These tests, with the same technology and simplicity of home pregnancy tests, brought about the potential to test anywhere and anytime; they were simple to use, cheap, reliable, and required little training or laboratory experience. They became so successful that they allowed for decentralization and task shifting: HIV testing in LMICs is now often performed by "expert" patients or health care workers who have been specifically trained to carry out POC HIV testing. ${ }^{11,12}$ More recent versions of these POC tests seek to shorten the window between infection and antibody production by using a combination of antibody and p24 antigen detection, and they have been used with some success in laboratory validation tests, but with only limited accuracy in clinical field settings in Malawi and the UK. ${ }^{13}$

There is one exception to the utility of POC HIV tests in LMICs. Maternal antibodies to HIV are transferred to the fetus through the placenta and continue to be present for up to 18 months after birth. Any antibody test would identify the infant of an HIV-infected woman as HIV-antibody positive, and so direct pathogen detection is required in infants up to 2 years of age. These tests are carried out by reverse transcriptase polymerase chain reaction (PCR)-based tests, which are expensive and not widely available in many LMICs. 
The development of future POC viral DNA tests that could potentially identify infected infants will be of enormous value in sub-Saharan Africa, where there are still 230,000 $\mathrm{HIV}^{+}$babies born each year. ${ }^{14}$ At present, infant testing is not well accessed, requiring heel-prick spot blood samples that necessitate complex expensive laboratory tests for viral DNA, with per-test costs estimated around USD20 and above. ${ }^{15}$ Estimates indicate that the take-up of these early infant diagnosis tests is between $6 \%$ and $15 \%$ in children up to 1 year old. ${ }^{16}$

\section{ART}

The modern era of ART has delivered highly effective regimens that, when used in combination, have been extremely successful in suppressing viral load (VL) and extending survival. ${ }^{17-19}$ Life expectancy for those living with HIV in high-income countries is approaching that of $\mathrm{HIV}^{-}$individuals, and there is increasing evidence of the positive impact of ART on life expectancy in resource-limited settings. ${ }^{20,21}$ The success of ART in the prevention of mother-to-child transmission (MTCT) has been dramatic. Prior to ART, around $25 \%-40 \%$ of infants became infected by HIV from their mother, usually around birth or through breast-feeding. ${ }^{22,23}$ Even a one-off dose of nevirapine near birth can reduce the risk of infection, and continued use of highly active ART during pregnancy and breast-feeding have resulted in MTCT rates of $<1 \% .{ }^{24-26}$ Finally, there is increasing interest in the use of ART as prevention, particularly in serodiscordant couples, and even at community or population levels. ${ }^{27,28}$

\section{Monitoring HIV: when to start treatment}

There are two other main diagnostic tests that are used specifically in HIV care: CD4 counts and HIV VL, the quantitation of HIV nucleic acid. CD4 counting is not, strictly speaking, a diagnostic test, but rather a flow cytometry research tool that was applied to count CD4 T-cells. Current national and international guidelines ${ }^{29}$ largely recommend initiation of ART based on a CD4 count. With the advent of better ART regimens, lower pill burdens, reduced toxicity, and increased barriers to viral resistance, the CD4 count threshold to start ART was increased to 350 cells $/ \mathrm{mm}^{3}$ and is likely to change to 500 cells $/ \mathrm{mm}^{3}$ in $2013 .{ }^{29}$ In high-income countries, there is a trend to initiate ART with ever-increasing CD4 count thresholds as the benefit of ART to prevent transmission of $\mathrm{HIV}$ is becoming increasingly apparent. A large, prospective cohort analysis published in 2010 demonstrated that viral suppression with ART for an $\mathrm{HIV}^{+}$individual reduces the risk of onward viral transmission to their sexual partners by $96 \%{ }^{27}$ Mathematical models show that universal HIV testing with immediate ART for all those found to be $\mathrm{HIV}^{+}$ has the potential to eliminate onward viral transmission at a population level. ${ }^{30-32}$ Current WHO recommendations for HIV serodiscordant couples recommend ART initiation irrespective of CD4 count. ${ }^{33}$ In this context, CD4 counting will no longer be necessary to guide ART initiation and all $\mathrm{HIV}^{+}$patients will begin ART when diagnosed, irrespective of CD4 T-cell counts. ${ }^{34,35}$

PCR-based techniques allowed for the detection, amplification, and quantitation of viral RNA in plasma, and the first US Food and Drug Administration-approved VL was approved in $1999^{36}$ for the Roche AMPLICOR system (Roche Molecular Systems Inc, Pleasanton, CA, USA). These tests, and others that employ nucleic acid amplification, have quickly become the gold standard for measurement and they are used mainly to monitor the response to ART. Failure to suppress the VL to undetectable levels often indicates poor adherence to the treatment regimen or the presence of viral resistance, necessitating a switch in therapy.

\section{When to start ART in LMICs}

In the absence of CD4-counting in LMICs, many start ART only when considered to be symptomatic with an AIDS-defining illness. However, many patients remain asymptomatic despite CD4 counts which, if measured, would trigger ART initiation. ${ }^{37,38}$ Patients are then denied ART but are at high risk of potentially fatal opportunistic infections. Syndromic management has proven to be inaccurate in determining ART eligibility, particularly in pregnant women, and, until the algorithms are improved, this strategy continues to be suboptimal for patient outcomes and the prevention of MTCT. ${ }^{39,40}$

\section{Tools used to monitor ART in high-income countries}

In high-income settings, the mainstay for ART monitoring are VL tests, CD4 counts, and an array of other diagnostics including liver function tests, renal function, and whole-blood counts. ${ }^{41}$ There is little evidence base for the frequency of these tests, but they are often performed quarterly. Newly diagnosed patients receive a CD4 count and VL, liver function tests, and renal function as estimated glomerular filtration rate (eGFR), as well as other sexual health screening. When a patient has started ART, there is an array of diagnostic tests performed regularly. Arguably, the most important of these is $\mathrm{VL}$, as it indicates (1) response to treatment, (2) emergence 
of drug-resistant virus, and (3) poor adherence to the ART regimen.

Patients successfully taking ART would expect to have "undetectable" virus levels (usually $<20-50$ copies HIV $\mathrm{RNA} / \mathrm{mL}$ ) in the plasma after 6-9 months of treatment. Failure to adhere to therapy is most often detected by the presence of detectable ( $>50$ copies HIV RNA $/ \mathrm{mL}$ ) viral replication in the presence of ART. Treatment adherence counseling to ensure the patient is treatment compliant may be sufficient to return the patient to the undetectable state. However, if persistent viral replication occurs on ART, then the patient has likely developed resistant virus and a switch in ART will be recommended. Liver function tests and eGFR are carried out to ensure that ART side effects, if any, are identified and alternative treatment regimens implemented.

Resistance testing is recommended in patients who are not fully suppressed or who have a suboptimal response to ART. ${ }^{41}$ Typically, genotypic testing is carried out before starting ART to identify mutations in the HIV genome that would render first-line treatment less efficacious and thus aid the selection of appropriate drug. This involves sequencing the viral genome to identify nucleotide mutations in key enzyme sequences. Phenotypic testing is often carried out when patients under treatment present with increased VL, and is an assay that measures viral replication in cell culture. Both assays are relatively expensive and results can take up to 2 weeks. Genotypic testing is often preferred due to its reduced time and cost. ${ }^{42}$

The recommendations for monitoring ART in LMICs are similar to those in high-income countries. VL measurement is the mainstay to identify viral replication. The WHO advise ${ }^{29}$ that $\mathrm{VL}$ levels of $>5,000$ copies $/ \mathrm{mL}$ indicate persistent treatment failure, although these levels would not be tolerated in high-income countries where a VL of $>200$ copies $/ \mathrm{mL}$ is considered a loss of virological control. In the absence of VL testing availability, immunological monitoring is considered to indicate treatment failure, although falls in CD4 count correlate less well with virological failure. ${ }^{43-45}$ In the absence of VL and CD4, syndromic management is again used to indicate the need for treatment switching. Resistance testing is not commonly performed in resource-poor settings for multiple reasons: there is little infrastructure, the assays are expensive, and, importantly, a lack of choice of second- or third-line therapy means that, despite the detection of viral resistance, there may not be any switch in therapy. Resistance testing is unlikely to be introduced in resource-poor settings in the near future.

\section{Logistical issues relating to HIV infection monitoring and impact on effective management of HIV}

Logistical issues in HIV monitoring are more pressing in LMICs, although there are issues in high-income countries with socialized medicine around increasing costs and patient experience. For the purpose of this review, we will focus on issues in LMICs.

\section{Logistical issues in LMICs}

The introduction of CD4 counting and VL testing would likely have a positive impact on the HIV pandemic in LMICs. Currently, these technologies are not cost-effective in these settings. ${ }^{46}$ The main barriers to monitoring HIV patients under treatment in LMICs are finances and infrastructure; both are clearly connected but can present distinct problems. The financial pressures to provide HIV care are enormous, with costs for ART around USD200 per person per year. ${ }^{47,48}$ In countries where the annual health care spend per capita is USD30, it is clear that HIV care can take up a significant portion of the country's health care budget (Table 2).

However, it is not solely a lack of budget that prevents the widespread introduction of CD4 and VL diagnostics into LMICs. Instrument purchase is a significant capital outlay and subsequent machine maintenance is also challenging. There is little infrastructure to support such diagnostics, instruments (if available) are more likely to be in disrepair, reagent supply is not guaranteed, and stock-outs are common. There are few trained staff to support quality CD4 and VL results and, of course, the demand for tests quickly overcomes the supply. VL measurement in particular is scarce due to the very high instrument costs, test complexity, and per-test price. Flow cytometers are complex instruments that require trained technical staff for operation and regular maintenance. Even if money were provided to purchase the reagents for CD4 counting, without significant infrastructure support, these instruments are unlikely to produce credible diagnostic results.

Table 2 Total expenditure (USD) per capita, per year on health care (20I I data)

\begin{tabular}{lll}
\hline Country & $\begin{array}{l}\text { Per capita/year total } \\
\text { health care spend }\end{array}$ & $\begin{array}{l}\text { HIV } \\
\text { prevalence }\end{array}$ \\
\hline USA & 8,607 & $0.7 \%$ \\
UK & 3,608 & $0.3 \%$ \\
New Zealand & 3,666 & $0.1 \%$ \\
Tanzania & 37 & $5.8 \%$ \\
Malawi & 31 & $10 \%$ \\
\hline
\end{tabular}

Note: Reproduced with permission from World Health Organization. HIVIAIDS: data and statistics. Available from: http://www.who.int/hiv/data/en/index.html. Accessed April 26, 2013.' 
A critical logistical issue that is apparent in many areas of LMICs is the absence of referral systems. The cascade of delivery of care spans from uptake of HIV testing to attendance at a local health care facility for repeat testing and CD4 counting, which necessitates return for test results and potential ART initiation. Significant proportions of people living with HIV are lost from care at each step. ${ }^{49-51}$ Health system strengthening with better linkage to care is desperately required in order to overcome many of these logistical blocks in LMICs.

The quandary for many ministries of health is that it was understood that using these diagnostic tools improved patient outcomes, but the costs of the tests were prohibitive (even before the costs of instrument purchase, staff, maintenance, and infrastructure support are added in).

\section{Logistical issues in high-income countries}

In high-income countries, there are increasing issues with patient satisfaction/logistics and costs. Quarterly blood testing requires the patient to attend the clinic twice - once to give the blood sample and the second time for the results. Clearly, this represents a significant cost to both the patient and the health service. It remains unclear if the current model of quarterly testing in high-income countries is sustainable or indeed preferable. CD4 counts and VL assays are similarly priced in well-resourced settings and comprise a large proportion of diagnostic budgets. Increasingly, clinics are moving toward 6-monthly monitoring for stable patients with only annual CD4 needed where sensitive VL assays are available.

\section{New developments in POC testing for HIV}

The successful implementation of ART in LMICs led to a drive to implement companion diagnostics such as CD4 and VL testing. At the same time, ART delivery was decentralized out of the large, urban hospitals to smaller health centers. This was critical, as it allowed ART to reach those who could not attend urban clinics due to ill health or simply because the paucity of transport options prohibited travel. Task shifting had had major implications for the delivery of HIV-related diagnostics and monitoring ART, as these diagnostics were to be performed in health care centers without trained laboratory staff. In settings where blood samples are sent to centralized laboratories for CD4 counts, the loss to follow-up is unacceptably high. ${ }^{49}$ In these cases, over half of the patients do not return to receive the result and are then lost to care, likely only to reenter the health system when significantly sicker/symptomatic. Not only is this a significant waste of resources for health systems that can scantly afford it, it may have disastrous consequences for patients who may not know that they desperately need treatment. These issues have driven development of POC tests and were instrumental in shaping the target product profile or ideal characteristics of the test. ${ }^{52}$ The last 10 years have seen a push to develop POC versions of laboratory tests frequently used in HIV care. ${ }^{53}$ The WHO issued recommendations for ideal characteristics for diagnostics used in LMICs. These criteria indicate that diagnostics should be Affordable, Sensitive, Specific, User-friendly, Robust and rapid, Equipment-free, and Deliverable (ASSURED) ${ }^{54}$ to those in need.

\section{POC CD4 tests}

The lack of CD4 counting facilities has two effects: patients denied ART because physicians wait for CD4 counts to be available (as per national guidelines), or CD4 counting was removed as the indicator for treatment initiation. The latter strategy was successfully used in countries like Malawi as a way to implement widespread ART. CD4 counting had long been the gateway to ART initiation in high-income countries and starting ART based on CD4 counts, rather than on syndromic management, is more accurate and leads to better outcomes for patients. ${ }^{55}$

The first wave of POC CD4 counting instruments were smaller and simpler versions of flow cytometers. Companies such as Guava (Millipore, Billerica, MA, USA) and Partec (Gorlitz, Germany) created "no-flow cytometers," which were more portable and required less infrastructure support than the lab-bound counterparts. However, these types of instruments had limited reach in LMICs, as they required complex software to produce the test result and so were relatively expensive. As with lab-based CD4 counts, they also need trained, dedicated operators. ${ }^{56}$

In 2005, the CD4 Initiative was created to promote and fund the development of new, POC versions of CD4 tests which were specifically aimed at LMIC markets. ${ }^{57}$ The Initiative aimed to look anew at POC CD4 technology and try to foster the development of simple, instrument-free POC CD4 tests that were appropriate for the setting in which they were to be used. Two new CD4 count tests have reached a commercialization stage from the Initiative's portfolio; both tests are truly POC and require limited laboratory infrastructure. The Burnet Institute test (now licensed as Vitisect ${ }^{\mathrm{TM}} \mathrm{CD} 4$, Omega Diagnostics Group, Alva, Scotland) is a simple, lateral flow assay that delivers a yes/no signal for treatment based 
on a threshold of 350 cells $/ \mathrm{mm}^{3}$. The assay can be performed without the need for electronic instrumentation, although the addition of an electronic reader allows for improved sensitivity and specificity but comes with significant instrument costs. Zyomyx, Inc (Fremont, CA, USA) developed a CD4 test that assembled established technology in a novel way. Unlike the Burnet test, it produces a quantitative CD4 count. It does not use electronic instrumentation but rather a mechanical spinner which is used to reduce the test time to under 10 minutes. The price per test is expected to be around USD8-USD10 and the spinner is expected to cost less than USD200. However, both of these tests are yet to undergo clinical trials in LMICs.

Alere (Cologne, Germany) developed a POC system, called PIMA, which provides CD4 counts using a finger-prick of blood. To date, this is the only commercially available POC CD4 counting system that has been extensively evaluated in high- and low-income settings to mostly positive outcomes. ${ }^{58,59}$ The instrument is relatively expensive, although not as much as a standard flow cytometer. It has a low throughput (2-3 samples per hour) with a test price of around USD10. Despite the success of Alere's PIMA CD4, there is still a need for simpler, higher-throughput POC CD4 tests for use in health care settings with limited infrastructure. The pipeline for POC CD4 testing is relatively rich, with several test developments expected to complete over the next 1 to 3 years. The more developed of these include systems from mBio (Washington, DC, USA) and the FACSPresto ${ }^{\mathrm{TM}}(\mathrm{BD}$, Franklin Lakes, NJ, USA), for which early evaluation data look promising. ${ }^{60}$

\section{POCVL}

In contrast to POC CD4, progress toward POC VL tests has been limited due to the sizeable technological hurdle represented by transferring nucleic acid amplification to a POC format. Developing a truly POC test with sensitivity limits like those of PCR is unlikely and possibly unnecessary for management of ART in the field, particularly in LMICs. Another barrier to successful POC VL development was the lack of consensus around when to switch ART in LMICs. With limited second-line treatment options, the benefits of switching treatment at very low HIV RNA copy numbers are not clear. The WHO guidelines suggest that VL levels above 5,000 copies/mL indicate treatment failure in these settings, but, in high-income countries with multiple treatment options, treatment switching occurs at RNA copy number in the low hundreds. ${ }^{29}$ PCR-based nucleic acid amplification techniques are used to detect very low copy numbers, but this technology is difficult to implement in LMICs. Other nucleic acid amplification techniques such as nucleic acid sequence-based amplification may be more appropriate for LMIC settings, but may not deliver low limits of detection used in high-income countries. A recent mathematical modeling study showed increasing cost-effectiveness of VL testing at higher thresholds $(>1,000$ copies/mL), although overall cost-effectiveness of VL monitoring compared to other strategies depends upon many complex and interacting factors. ${ }^{61}$

These factors and limitations are critical in shaping the target product profile of a POC VL test and the feasibility of a diagnostics development program. Higher switching thresholds may allow simpler technology to be incorporated into the POC VL test and so will reduce costs, development time, and the final test complexity and need for expensive instrumentation. Similar arguments can be made for the target sensitivity and specificity of a POC VL test in LMICs where lower targets ( $<90 \%$ sensitivity and specificity) may still have enormous benefits, particularly for early infant diagnosis, where direct pathogen detection is required and quantitation of VL is not necessary. ${ }^{62}$ The CD4 Initiative ${ }^{57}$ set out very clear specifications, or a target product profile (TPP), for POC CD4 tests in 2006. An ideal TPP for POC $\mathrm{VL}$ is emerging and we have set out our vision of a POC VL that will have a positive impact on HIV/AIDS care in LMICs (Table 3).

Currently, there are no commercially available POC VL systems, although, as with CD4, the pipeline is rich with emerging candidates, some of which have published very encouraging preliminary evaluation data. ${ }^{63,64}$

However, these candidate instruments are not truly POC, but could be used in laboratories where sufficient infrastructure support would permit quality VL results to be produced. Alere's Q system is scheduled to be completed in the next 1-2 years as is Diagnostics for the Real World's SAMBA EID/VL (Sunnyvale, CA, USA) and IQuum's Liat system (Marlborough, MA, USA). The reach of these tests into LMICs, particularly into those areas where the least infrastructure support is available, may be limited by the high instrument costs and a per-test price of around USD20. A truly POC VL test is still some years away, although the need for VL testing is growing as the availability of secondline drug therapies in LMICs increases. Finally, a large, randomized control trial, the Delivery of Antiretroviral Therapy in Africa (DART), demonstrated that participants who did not receive diagnostic laboratory monitoring but had frequent and detailed clinical monitoring had equivalent outcomes to those who received diagnostic results such as CD4 
Table 3 A target product profile for a point-of-care viral load test for use in low- and middle-income countries

\begin{tabular}{|c|c|c|c|}
\hline & Minimum specification & Optimal specification & Comments and key hurdles \\
\hline Threshold & $\begin{array}{l}5,000 \text { copies } / \mathrm{mL} \text { (as per } \mathrm{WHO} \\
\text { recommendation) }\end{array}$ & $\mathrm{I}, 000$ copies $/ \mathrm{mL}$ & Dependent upon NAAT chosen \\
\hline $\begin{array}{l}\text { Quantitative } \\
\text { or qualitative }\end{array}$ & $\begin{array}{l}\text { Qualitative or semi-quantitative } \\
\text { (yes/no answer for treatment switching } \\
\text { or pathogen detection in EID) }\end{array}$ & $\begin{array}{l}\text { Fully quantitative with } \\
\text { a LOD of } 200-500 \text { copies }\end{array}$ & Again dependent upon NAAT \\
\hline Time to result & $<2$ hours & $<$ I hours & $\begin{array}{l}\text { May be some flexibility depending upon } \\
\text { ability to batch test }\end{array}$ \\
\hline Throughput & Minimum of 8 samples per day & $>25$ tests/operator/day & $\begin{array}{l}\text { Dependent on test complexity and batch } \\
\text { testing }\end{array}$ \\
\hline Sample source & Capillary blood & Capillary blood & $\begin{array}{l}\text { If test is POC then no need for sample } \\
\text { transport: dried blood spot to be avoided } \\
\text { Limited access to phlebotomy services } \\
\text { in LMIC }\end{array}$ \\
\hline Test simplicity & No more than 5 steps & Fewer than 3 steps & \\
\hline $\begin{array}{l}\text { Instrumentation } \\
\text { specification }\end{array}$ & $\begin{array}{l}\text { Small instrument acceptable } \\
\text { Limited software/complexity } \\
\text { Must operate on battery power at minimum } \\
\text { Limited infrastructure support needed } \\
\text { Potential to be used in mid-level health centers/ } \\
\text { district general hospitals } \\
\text { Maintenance model must be supportable }\end{array}$ & $\begin{array}{l}\text { No instrumentation required } \\
\text { No infrastructure required } \\
\text { Can be used in simple health } \\
\text { centers } \\
\text { No AC/DC power needed }\end{array}$ & $\begin{array}{l}\text { Difficult optimal specification. Currently } \\
\text { no technology for VL detection which is } \\
\text { instrument-free, nor in the development } \\
\text { pipeline }\end{array}$ \\
\hline Test performance & $>90 \%$ sensitivity and specificity & $\begin{array}{l}>95 \% \text { sensitivity } \\
\text { and specificity }\end{array}$ & \\
\hline Stability & $40^{\circ} \mathrm{C}$ with high humidity for 12 months & $\begin{array}{l}40^{\circ} \mathrm{C} \text { with high humidity } \\
\text { for } 18-24 \text { months }\end{array}$ & $\begin{array}{l}\text { Closed vial stability } \\
\text { Dependent on transport logistics }\end{array}$ \\
\hline $\begin{array}{l}\text { Target manufacture } \\
\text { price (USD) (test) }\end{array}$ & $\$ 10$ & $<\$ 5$ & $\begin{array}{l}\text { Distribution costs can double the test } \\
\text { price, depending on distribution models } \\
\text { Exclusive of required quality assurance } \\
\text { materials }\end{array}$ \\
\hline $\begin{array}{l}\text { Target manufacture } \\
\text { price (instrument) }\end{array}$ & $<\$ 8,000$ & $<\$ 1,000$ if required & Must include maintenance \\
\hline $\begin{array}{l}\text { Additional materials } \\
\text { (excluding qa } \\
\text { materials) }\end{array}$ & None: everything must be included & None & $\begin{array}{l}\text { If water and power supplies are variable, } \\
\text { not feasible to ask for buffers or other } \\
\text { instruments and materials }\end{array}$ \\
\hline Disposal & Safe solution of contaminated materials & $\begin{array}{l}\text { Safe solution } \\
\text { of contaminated } \\
\text { materials }\end{array}$ & $\begin{array}{l}\text { Burning most common method } \\
\text { Ensure sufficient accelerant in the test } \\
\text { materials for complete incineration }\end{array}$ \\
\hline
\end{tabular}

Abbreviations: EID, early infant diagnosis; LMICs, low- and middle-income countries; POC, point-of-care; VL, viral load; WHO, World Health Organization; LOD, limit of detection; NAAT, nucleic acid amplification technique; qa, quality assurance; $A C / D C$, alternating current/direct current.

and VL. ${ }^{65}$ These data suggest that, when using a preferred high-income country ART regimen that includes tenofovir, diagnostic testing may not be cost-effective at current prices. ${ }^{46}$ However, this study implemented a high level of clinical care for an African environment that is not widely found or currently implementable. The follow-up study, LabLite, ${ }^{66}$ is examining the use of clinical algorithms in the management of HIV patients in LMICs. However, DART did not examine the impact of the cost-effectiveness of POC testing in these settings. It is likely that the lower costs of POC tests will drive the cost-effectiveness of CD4 and VL use in LMICs. ${ }^{67}$

\section{Impact of POC CD4 tests on ART}

To date, there are no POC VL tests commercially available and so their impact on HIV care and ART cannot yet be assessed. POC CD4 systems have been commercially available for 2 or 3 years and so data are accumulating on their impact on HIV/AIDS care and patient outcomes. POC CD4 tests are likely to have a positive impact on several points on the care pathway:

1. Retention to care in ART eligible patients.

2. Increasing access to ART and thus improving overall survival and increasing treatment efficacy.

3. Reducing time to ART from diagnosis.

4. Reducing MTCT.

In a study of mobile HIV counseling and testing in South Africa, patients were pseudorandomized to receive a POC CD4 test following HIV testing or the normal standard of care, which was referral to another clinic with flow cytometric CD4 counting. Sixty-two percent of patients who had 
received a POC CD4 test at diagnosis had completed their referral visit within 8 weeks, compared to $47 \%$ of those who had not received the POC CD4 test. ${ }^{68}$

Similarly, a comprehensive study in Mozambique demonstrated that the use of PIMA POC CD4 in primary care clinics had multiple effects on retention to care. Of those enrolled, $78 \%$ of patients received CD4 count staging within 90 days using the POC CD4 test, compared to $42 \%$ of those with conventional care. The use of POC CD4 tests resulted in 94 patients initiating ART within 60 days of CD4 staging, compared to 57 in the standard-of-care arm. ${ }^{69}$ The same study demonstrated that introduction of POC CD4 tests led to a reduction in the number of days from enrollment to ART initiation of 48 to 20 days. POC CD4 test implementation also decreased the loss to follow-up, reducing it from $64 \%$ to $33 \%$. Overall, the number of patients enrolled in the study who initiated ART increased to $22 \%$ from $12 \%$ without POC CD4. ${ }^{69}$

ART-eligible pregnant women are at high risk of transmitting the virus to their infant. A study in Zimbabwe found that the introduction of POC CD4 testing increased the number of pregnant women receiving CD4 counts from 617 with standard-of-care to 890 with POC CD4 testing. Crucially, POC CD4 testing increased the proportion of pregnant women initiated on ART from $9 \%$ to $25 \%{ }^{70}$

In another study in Zimbabwe, eligible $\mathrm{HIV}^{+}$patients visit the treatment center three times before accessing ART. The introduction of POC CD4 testing reduced the number of clinic visits to two. Equally importantly, implementing POC CD4 testing reduced the time to ART from 60 days with standard-of-care to 24 days with POC CD4. ${ }^{71}$

The cost-effectiveness of POC CD4 testing has also been examined. Several studies have also indicated that POC CD4 testing is cost-effective when compared to standard-of-care (flow cytometry) or syndromic management. ${ }^{67,72}$ These types of analyses are increasingly important to justify the investment of POC CD4 testing and its impact on the health care system and population.

\section{Conclusion}

The introduction of ART has revolutionized the HIV pandemic. Thanks to an unprecedented global health care effort, there are now fewer new HIV infections than 10 years ago. This effort would not have been possible without the use of diagnostics tests, either for diagnosis or for the initiation and monitoring of patients on ART. Due to the high costs of these tests and the relative complexity of these assays, they have been difficult if not impossible to implement widely in resource-poor settings. Lack of infrastructure, money, and trained staff and poor referral/health systems have prevented widespread uptake of the traditional diagnostics found in high-income country laboratories. Consequently, there has been a drive to develop new and more practical POC tests that are designed specifically for the settings in which they are to be used. Programs like the CD4 Initiative were specifically created to drive the development of new, innovative CD4 tests, and major players in the diagnostics have since entered into this market space. The impact of POC CD4 tests on ART is only just emerging, but studies report improved retention to care and reduced loss to follow-up in LMICs. In terms of onward transmission risk in prevention of MTCT programs, low-cost POC VL testing has the potential to dramatically alter patient management in all settings and may supersede the need for CD4 testing. In high-income countries, POC tests will improve patient experience and, assuming the tests are priced accordingly, may be a cost-effective solution to the increasing costs involved in HIV care.

\section{Acknowledgment}

The authors would like to acknowledge funding from The Monument Trust and the BRC of Imperial College NHS Trust.

\section{Disclosure}

The authors report no conflicts of interest in this work.

\section{References}

1. HIV/AIDS: data and statistics [webpage on the Internet]. Geneva: World Health Organization; 2013. Available from: http://www.who.int/hiv/ data/en/index.html. Accessed April 26, 2013.

2. Barré-Sinoussi F, Chermann JC, Rey F, et al. Isolation of a T-lymphotropic retrovirus from a patient at risk for acquired immune deficiency syndrome (AIDS). Science. 1983;220(4599):868-871.

3. Gallo RC, Salahuddin SZ, Popovic M, et al. Frequent detection and isolation of cytopathic retroviruses (HTLV-III) from patients with AIDS and at risk for AIDS. Science. 1984;224(4648):500-503.

4. Sarngadharan MG, Popovic M, Bruch L, Schupbach J, Gallo RC. Antibodies reactive with human T-lymphotropic retroviruses (HTLV-III) in the serum of patients with AIDS. Science. 1984;224(4648):506-508.

5. Petricciani JC. Licensed tests for antibody to human T-lymphotropic virus type III. Sensitivity and specificity. Ann Intern Med. 1985;103(5): 726-729.

6. Schorr JB, Berkowitz A, Cumming PD, Katz AJ, Sandler SG. Prevalence of HTLV-III antibody in American blood donors. $N$ Engl $J$ Med. 1985;313(6):384-385.

7. Biggar RJ. The AIDS problem in Africa. Lancet. 1986;1(8472): 79-83.

8. Downing RG, Otten RA, Marum E, et al. Optimizing the delivery of HIV counseling and testing services: the Uganda experience using rapid HIV antibody test algorithms. J Acquir Immune Defic Syndr Hum Retrovirol. 1998;18(4):384-388.

9. Pai NP, Tulsky JP, Cohan D, Colford JM Jr, Reingold AL. Rapid point-of-care HIV testing in pregnant women: a systematic review and meta-analysis. Trop Med Int Health. 2007;12(2):162-173.

10. HIV Assays: Operational Characteristics (Phase 1). Report 14: Simple/ Rapid Tests. Geneva: World Health Organization; 2004. 
11. Cohen R, Lynch S, Bygrave H, et al. Antiretroviral treatment outcomes from a nurse-driven, community-supported HIV/AIDS treatment programme in rural Lesotho: observational cohort assessment at two years. J Int AIDS Soc. 2009; 12:23.

12. Bemelmans M, van den Akker T, Ford N, et al. Providing universal access to antiretroviral therapy in Thyolo, Malawi through task shifting and decentralization of HIV/AIDS care. Trop Med Int Health. 2010; 15(12):1413-1420.

13. Chetty V, Moodley D, Chuturgoon A. Evaluation of a 4th generation rapid HIV test for earlier and reliable detection of HIV infection in pregnancy. J Clin Virol. 2012;54(2):180-184.

14. Wessman MJ, Theilgaard Z, Katzenstein TL. Determination of HIV status of infants born to HIV-infected mothers: a review of the diagnostic methods with special focus on the applicability of p24 antigen testing in developing countries. Scand J Infect Dis. 2012;44(3):209-215.

15. Ciaranello AL, Park JE, Ramirez-Avila L, Freedberg KA, Walensky RP, Leroy V. Early infant HIV-1 diagnosis programs in resource-limited settings: opportunities for improved outcomes and more cost-effective interventions. BMC Med. 2011;9:59.

16. Towards universal access: scaling up priority HIV/AIDS interventions in the health sector: progress report 2010. Geneva: World Health Organization; 2010.

17. Gallant JE, Staszewski S, Pozniak AL, et al. Efficacy and safety of tenofovir DF vs stavudine in combination therapy in antiretroviral-naive patients: a 3-year randomized trial. JAMA. 2004;292(2):191-201.

18. Gallant JE, DeJesus E, Arribas JR, et al; Study 934 Group. Tenofovir DF, emtricitabine, and efavirenz vs zidovudine, lamivudine, and efavirenz for HIV. N Engl J Med. 2006;354(3):251-260.

19. Clotet B, Bellos N, Molina JM, et al; POWER 1 and 2 study groups Efficacy and safety of darunavir-ritonavir at week 48 in treatmentexperienced patients with HIV-1 infection in POWER 1 and 2: a pooled subgroup analysis of data from two randomised trials. Lancet. 2007; 369(9568):1169-1178.

20. van Sighem AI, Gras LA, Reiss P, Brinkman K, de Wolf F; ATHENA national observational cohort study. Life expectancy of recently diagnosed asymptomatic HIV-infected patients approaches that of uninfected individuals. AIDS. 2010;24(10):1527-1535.

21. Tanser F, Barnighausen T, Grapsa E, Zaidi J, Newell ML. High coverage of ART associated with decline in risk of HIV acquisition in rural KwaZulu-Natal, South Africa. Science. 2013;339(6122):966-971.

22. Datta P, Embree JE, Kreiss JK, et al. Mother-to-child transmission of human immunodeficiency virus type 1: report from the Nairobi Study. J Infect Dis. 1994;170(5):1134-1140.

23. [No authors listed]. Mother-to-child transmission of HIV infection. The European Collaborative Study. Lancet. 1988;2(8619):1039-1043.

24. Stringer JS, Rouse DJ, Sinkala M, et al. Nevirapine to prevent mother-to-child transmission of HIV-1 among women of unknown serostatus. Lancet. 2003;362(9398):1850-1853.

25. Lallemant M, Jourdain G, Le Coeur S, et al. Single-dose perinatal nevirapine plus standard zidovudine to prevent mother-to-child transmission of HIV-1 in Thailand. N Engl J Med. 2004;351(3):217-228.

26. Dryden-Peterson S, Jayeoba O, Hughes MD, et al. Highly active antiretroviral therapy versus zidovudine for prevention of mother-to-child transmission in a programmatic setting, Botswana. J Acquir Immune Defic Syndr. 2011;58(3):353-357.

27. Donnell D, Baeten JM, Kiarie J, et al; Partners in Prevention HSV/ HIV Transmission Study Team. Heterosexual HIV-1 transmission after initiation of antiretroviral therapy: a prospective cohort analysis. Lancet. 2010;375(9731):2092-2098.

28. Zaidi J, Grapsa E, Tanser F, Newell ML, Barnighausen T. Dramatic increases in HIV prevalence after scale-up of antiretroviral treatment: a longitudinal population-based HIV surveillance study in rural kwazulu-natal. AIDS. Epub May 10, 2013.

29. WHO. Consolidated guidelines on the use of antiretroviral drugs for treating and preventing HIV infection: recommendations for a public health approach. Available from http://www.who.int/hiv/pub/guidelines/ arv2013/download/en/. Accessed August 1, 2013.
30. Celum C, Hallett TB, Baeten JM. HIV-1 prevention with ART and PrEP: mathematical modeling insights into resistance, effectiveness, and public health impact. J Infect Dis. 2013;208(2): 189-191.

31. Granich R, Crowley S, Vitoria M, et al. Highly active antiretroviral treatment for the prevention of HIV transmission. J Int AIDS Soc. 2010;13:1.

32. Granich RM, Gilks CF, Dye C, De Cock KM, Williams BG. Universal voluntary HIV testing with immediate antiretroviral therapy as a strategy for elimination of HIV transmission: a mathematical model. Lancet. 2009;373(9657):48-57.

33. Guidance on couples HIV testing and counselling - including antiretroviral therapy for treatment and prevention in serodiscordant couples. Geneva: World Health Organization; 2012.

34. Cohen MS, Chen YQ, McCauley M, et al. Prevention of HIV-1 infection with early antiretroviral therapy. $N$ Engl J Med. 2011;365(6): 493-505.

35. Osborne BJ, Sheth PM, Yi TJ, et al. Impact of antiretroviral therapy duration and intensification on isolated shedding of HIV-1 RNA in semen. J Infect Dis. 2013;207(8):1226-1234.

36. US Food and Drug Administration. [webpage on the Internet] Available from www.fda.gov. Accessed July 22, 2013.

37. Carter RJ, Dugan K, El-Sadr WM, et al. CD4+ cell count testing more effective than HIV disease clinical staging in identifying pregnant and postpartum women eligible for antiretroviral therapy in resource-limited settings. J Acquir Immune Defic Syndr. 2010;55(3):404-410.

38. Torpey K, Lartey M, Amenyah R, et al. Initiating antiretroviral treatment in a resource-constrained setting: does clinical staging effectively identify patients in need? Int J STD AIDS. 2009;20(6):395-398.

39. Emmett SD, Cunningham CK, Mmbaga BT, et al. Predicting virologic failure among HIV-1-infected children receiving antiretroviral therapy in Tanzania: a cross-sectional study. J Acquir Immune Defic Syndr. 2010;54(4):368-375.

40. Baveewo S, Ssali F, Karamagi C, et al. Validation of World Health Organisation HIV/AIDS clinical staging in predicting initiation of antiretroviral therapy and clinical predictors of low CD4 cell count in Uganda. PLoS One. 2011;6(5):e19089.

41. Asboe D, Aitken C, Boffito M, et al; BHIVA Guidelines Subcommittee. British HIV Association guidelines for the routine investigation and monitoring of adult HIV-1-infected individuals 2011. HIV Med. 2012;13(1):1-44.

42. Tang MW, Shafer RW. HIV-1 antiretroviral resistance: scientific principles and clinical applications. Drugs. 2012;72(9):e1-e25.

43. Ferreyra C, Yun O, Eisenberg N, et al. Evaluation of clinical and immunological markers for predicting virological failure in a HIV/AIDS treatment cohort in Busia, Kenya. PLoS One. 2012;7(11):e49834.

44. Arnedo M, Alonso E, Eisenberg N, et al. Monitoring HIV viral load in resource limited settings: still a matter of debate? PLoS One. 2012;7(12): e47391.

45. Ingole N, Mehta P, Pazare A, Paranjpe S, Sarkate P. Performance of immunological response in predicting virological failure. AIDS Res Hum Retroviruses. 2013;29(3):541-546.

46. Medina Lara A, Kigozi J, Amurwon J, et al; DART Trial Team. Cost effectiveness analysis of clinically driven versus routine laboratory monitoring of antiretroviral therapy in Uganda and Zimbabwe. PLoS One. 2012;7(4):e33672.

47. Stover J, Korenromp EL, Blakley M, et al. Long-term costs and health impact of continued global fund support for antiretroviral therapy. PLoS One. 2011;6(6):e21048.

48. Boyer S, March L, Kouanfack C, et al; Stratall ANRS 12110/ESTHER Study Group. Monitoring of HIV viral load, CD4 cell count, and clinical assessment versus clinical monitoring alone for antiretroviral therapy in low-resource settings (Stratall ANRS 12110/ESTHER): a costeffectiveness analysis. Lancet Infect Dis. 2013;13(7):577-586.

49. Tayler-Smith K, Zachariah R, Massaquoi M, et al. Unacceptable attrition among WHO stages 1 and 2 patients in a hospital-based setting in rural Malawi: can we retain such patients within the general health system? Trans R Soc Trop Med Hyg. 2010;104(5):313-319. 
50. Govindasamy D, Ford N, Kranzer K. Risk factors, barriers and facilitators for linkage to antiretroviral therapy care: a systematic review. AIDS. 2012;26(16):2059-2067.

51. Govindasamy D, van Schaik N, Kranzer K, Wood R, Mathews C, Bekker LG. Linkage to HIV care from a mobile testing unit in South Africa by different CD4 count strata. J Acquir Immune Defic Syndr. 2011;58(3):344-352.

52. Zachariah R, Reid SD, Chaillet P, Massaquoi M, Schouten EJ, Harries AD. Viewpoint: why do we need a point-of-care CD4 test for low-income countries? Trop Med Int Health. 2011;16(1):37-41.

53. Laursen L. Point-of-care tests poised to alter course of HIV treatment. Nat Med. 2012;18(8):1156.

54. Peeling RW, Holmes KK, Mabey D, Ronald A. Rapid tests for sexually transmitted infections (STIs): the way forward. Sex Transm Infect. 2006;82(5):v1-v6.

55. Kitahata MM, Gange SJ, Abraham AG, et al. Effect of early versus deferred antiretroviral therapy for HIV on survival. $N$ Engl J Med. 2009;360(18):1815-1826.

56. Spacek LA, Shihab HM, Lutwama F, et al. Evaluation of a low-cost method, the Guava EasyCD4 assay, to enumerate CD4-positive lymphocyte counts in HIV-infected patients in the United States and Uganda. J Acquir Immune Defic Syndr. 2006;41(5):607-610.

57. The CD4 Initiative [webpage on the Internet]. London: Imperial College London; 2006. Available from: http://www3.imperial.ac.uk/ cd4. Accessed May 2, 2013.

58. Sukapirom K, Onlamoon N, Thepthai C, Polsrila K, Tassaneetrithep B, Pattanapanyasat K. Performance evaluation of the Alere PIMA CD4 test for monitoring HIV-infected individuals in resource-constrained settings. J Acquir Immune Defic Syndr. 2011;58(2):141-147.

59. Jani IV, Sitoe NE, Chongo PL, et al. Accurate CD4 T-cell enumeration and antiretroviral drug toxicity monitoring in primary healthcare clinics using point-of-care testing. AIDS. 2011;25(6):807-812.

60. Bornheimer S, Bui N, Le D, et al. Development of the BD FACSPresto instrument, a new point-of-care system for CD4+ counting. IAS 2013; June 30-July 3, 2013; Kuala Lumpur, Malaysia. Available at http://pag. ias2013.org/Abstracts.aspx?AID=1471. Accessed August 1, 2013.

61. Estill J, Egger M, Blaser N, et al; IeDEA Southern Africa. Cost-effectiveness of point-of-care viral load monitoring of ART in resource-limited settings: mathematical modelling study. AIDS. Epub March 4, 2013.

62. Urdea M, Penny LA, Olmsted SS, et al. Requirements for high impact diagnostics in the developing world. Nature. 2006;444 Suppl 1: 73-79.

63. Lee HH, Dineva MA, Chua YL, Ritchie AV, Ushiro-Lumb I, Wisniewski CA. Simple amplification-based assay: a nucleic acid based point-of-care platform for HIV-1 testing. Journal Infect Dis. 2010;201: S65-S72.
64. Jani I, Meggi B, Vubil A, et al. Evaluation of point-of-care nucleic acid testing for HIV viral load and early infant diagnosis in primary health clinics: Mozambique. Abstract presented at Conference on Retroviruses and Opportunistic Infections; 2013; Atlanta, GA. March 3-6, 2013.

65. DART Trial Team, Mugyenyi P, Walker AS, et al. Routine versus clinically driven laboratory monitoring of HIV antiretroviral therapy in Africa (DART): a randomised non-inferiority trial. Lancet. 2010;375(9709):123-131

66. Bongomera G, Namata H, Nkhata M, et al. Lablite: baseline mapping survey of decentralised ART service provision in Malawi, Uganda and Zimbabwe. Conference abstract. 19th International AIDS Conference; July 22-July 27, 2012; Washington DC.

67. Grundy C, Medina Lara A, Winogron D, et al. Point-of-care CD4 tests can increase life-years saved with reduced costs compared to flow cytometric CD4 counting. Abstract presented at the Sixth International AIDS Society Conference on HIV Pathogenesis, Treatment and Prevention; 2011; Rome.

68. Larson BA, Schnippel K, Ndibongo B, et al. Rapid point-of-care CD4 testing at mobile HIV testing sites to increase linkage to care: an evaluation of a pilot program in South Africa. J Acquir Immune Defic Syndr. 2012;61(2):e13-e17.

69. Jani IV, Sitoe NE, Alfai ER, et al. Effect of point-of-care CD4 cell count tests on retention of patients and rates of antiretroviral therapy initiation in primary health clinics: an observational cohort study. Lancet. 2011;378(9802):1572-1579.

70. Muchedzi A, Chadambuka A, Chikwinya B, Mahomva A. Evaluating the effect of the use of point-of-care CD4 machines on access to antiretroviral therapy (ART) eligibility screening and ART initiation for HIV-positive pregnant women in Zimbabwe: towards elimination of new paediatric HIV infection by 2015. J Int AIDS Soc. 2012;15:282-282. Abstract presented at IAS; July 22-27; 2012; Washington, DC, USA.

71. Hatzold K, Gudukeya SB, Museta M, et al. Improved access to HAART through point of care CD4 cell count testing integration into HIV testing and counseling services in Zimbabwe. 6th IAS Conference on HIV Pathogenesis and Treatment; 2011; Rome. Conference Abstract; July 17- July 20, 2011.

72. Ciaranello AL, Myer L, Christensen S, et al. The cost-effectiveness of point-of-care CD4 testing in antenatal care in South Africa. Abstract presented at Conference on Retroviruses and Opportunistic Infections; 2013; Atlanta, GA. March 3-6, 2013.
Clinical Epidemiology

\section{Publish your work in this journal}

Clinical Epidemiology is an international, peer-reviewed, open access journal focusing on disease and drug epidemiology, identification of risk factors and screening procedures to develop optimal preventative initiatives and programs. Specific topics include: diagnosis, prognosis, treatment, screening, prevention, risk factor modification, systematic

\section{Dovepress}

reviews, risk \& safety of medical interventions, epidemiology \& biostatical methods, evaluation of guidelines, translational medicine, health policies \& economic evaluations. The manuscript management system is completely online and includes a very quick and fair peer-review system, which is all easy to use. 